\title{
AVALIAÇÃO DE PARÂMETROS BIOMÉTRICOS, FISIOLÓGICOS E BIOQUÍMICOS EM PACIENTES COM SÍNDROME METABÓLICA SUBMETIDOS A PROGRAMA DE ATIVIDADE FÍSICA APÓS CIRURGIA BARIÁTRICA SANTORO II
}

\author{
EVALUATION OF BIOMETRIC, PHYSIOLOGICAL AND BIOCHEMICAL \\ PARAMETERS IN METABOLIC SYNDROME PATIENTS SUBMITTED \\ TO A PHYSICAL ACTIVITY PROGRAM AFTER \\ SANTORO II BARIATRIC SURGERY
}

\begin{abstract}
Kátia Cristina Alonso', Flávio Guimarães Kalinowski², Fábio Quirillo Milléo ${ }^{3}$, Roberto Ferreira Artoni ${ }^{4}$, Viviane Nogaroto ${ }^{4}$, Carlos Ricardo Maneck Malfatti ${ }^{5}$

1 Universidade Estadual de Ponta Grossa, Programa de Pós-Graduação em Biologia Evolutiva, Ponta Grossa, Paraná, Brasil. E-mail: <katiacalonso@hotmail.com>

2 Universidade Estadual de Ponta Grossa, Departamento de Educação Física, Ponta Grossa, Paraná, Brasil. E-mail: <kalinows@uepg.br>

3 Departamento de Cirurgia, Hospital Vicentino da Sociedade Beneficente São Camilo, Ponta Grossa, PR, Brasil. E-mail: <fabiomilleo@uol.com.br>

${ }^{4}$ Universidade Estadual de Ponta Grossa, Departamento de Biologia Estrutural, Molecular e Genética, Programa de Pós-Graduação em Biologia Evolutiva, Ponta Grossa, Paraná, Brasil.E-mail: <rfartoni@pq.cnpq.br>; <vivianenogaroto@hotmail.com>

5 Universidade Estadual do Centro-Oeste, Setor de Ciências da Saúde, Irati, Paraná, Brasil. E-mail: <ricardo.malfatti@bol.com.br>

* Corresponding author: Viviane Nogaroto. Departamento de Biologia Estrutural, Molecular e Genética. Laboratório de Citogenética e Evolução. Universidade Estadual de Ponta Grossa. Av. Carlos Cavalcanti, 4748, CEP 84030-900, Ponta Grossa, PR, Brasil. Tel.: +55 42 3220-3739; Fax: +55 42 3220-3102; E-mail: <vivianenogaroto@hotmail. com>
\end{abstract}

Recebido para publicação em 01/02/2011

Aceito para publicação em 10/04/2011

\section{RESUMO}

O estudo teve como objetivo investigar os resultados de um programa de atividade física em pacientes portadores de Síndrome Metabólica (SM) submetidos ao procedimento cirúrgico bariátrico denominado Santoro II. Um mês após a cirurgia, dois pacientes (um homem e uma mulher) foram submetidos a uma avaliação antropométrica, bem como a testes bioquímicos, antes de iniciarem atividade física prevista, composta de exercícios aeróbicos durante 30 minutos em cerca de 50\% da frequência cardíaca máxima, seguido de um treino de resistência anaeróbica e alongamentos de membros superiores e inferiores antes e após os exercícios físicos. 
Este programa se estendeu por nove meses, período em que foram realizados os testes. Os níveis de colesterol, LDL (Low Density Lipoprotein) e triglicérides diminuíram tanto no homem quanto na mulher, a qual também teve seus níveis de HDL (High Density Lipoprotein) aumentados. Os parâmetros biométricos diminuíram, levando a uma boa classificação do IMC (Índice de Massa Corporal) e RCQ (Relação Cintura-Quadril) ao final do programa, o mesmo ocorrendo no teste fisiológico. Assim, Santoro II, seguido por um programa de atividade física, mostrou ser eficiente no tratamento de pacientes portando SM, levando a uma melhora em todos os parâmetros analisados.

Palavras-chave: Obesidade. Exercícios aeróbicos. Dislipidemia. Qualidade de vida. Fatores de risco cardiovasculares.

\begin{abstract}
The study investigated the effect of a physical activity program in Metabolic Syndrome (MS) patients after bariatric surgery, called Santoro II. One month after surgery, two patients (a woman and a man) were submitted to anthropometric and biochemical tests before a physical activity program, which consisted of 30 minutes of aerobic exercises, about $50 \%$ of maximum heart rate, followed by an anaerobic resistance training, as well as stretching of arms and legs before and after exercise. The physical activity program was extended for 9 months, when the tests were performed. The levels of cholesterol, LDL (Low Density Lipoprotein) and triglycerides decreased in both man and woman, who also had HDL (High Density Lipoprotein) levels increased. After the exercises, the biometric parameters decreased, resulting in a better classification based on Body Mass Index (BMI) and waist-hip ratio (WHR), and the physiologic tests also improved. Thus, Santoro II followed by a physical activity program was effective in treating patients carrying MS leading to an improvement in all parameters analyzed in this study.
\end{abstract}

Keywords: Obesity. Aerobic Exercises. Dyslipidemia. Quality of Life.

Cardiovascular Risk Factors.

\section{Introdução}

O aumento da ingestão calórica diária e a diminuição de atividades físicas podem levar ao aumento da massa corporal e, consequentemente, à obesidade, condição considerada uma pandemia pela Organização Mundial da Saúde (WHO, 2000). O conjunto de comorbidades relacionadas à obesidade, como diabetes tipo 2 (DM2), dislipidemias (aumento nos níveis de LDL - Low Density Lipoprotein - e diminuição de HDL -
High Density Lipoprotein), obesidade abdominal e hipertensão arterial (GRUNDY et al., 2004; ISOMAA et al., 2001), levam a uma desordem metabólica denominada Síndrome Metabólica (SM). A etiologia da SM é complexa e caracteriza defeitos em diversos sistemas homeostáticos (HOPKINS et al., 1996). Uma série de definições para SM tem sido proposta (ALEXANDER et al., 2003), e neste estudo serão considerados os critérios do National Cholesterol Education Program (NCEP, 2002). Estudos indicam a alta prevalência 
de SM (acomete mais de 39\% de homens e $22 \%$ de mulheres) em populações adultas dos Estados Unidos, México e Finlândia (LAKKA et al., 2002; FORD, 2004).

Os fatores de risco de portadores de SM e a prática de atividade física regular mostram uma relação inversamente proporcional (HOPKINS et al., 1996; LAAKSONEN, 2002). Christian et al. (2008), estudando o efeito benéfico do exercício físico em diabéticos tipo 2 , verificaram o aumento da hemoglobina $\mathrm{A} 1 \mathrm{c}$, associado ao menor risco cardiovascular e mortalidade global, e ainda a melhoria do controle metabólico.

As cirurgias bariátricas têm sido empregadas como um recurso extremo para o controle da obesidade mórbida (Índice de Massa Corporal 35 $\mathrm{kg} / \mathrm{m}^{2}$ ) em casos onde tratamentos não cirúrgicos para perda de peso tiveram insucesso (NIH, 1991). A técnica cirúrgica de Santoro II, um procedimento inovador embasado em dados fisiológicos e antropológicos de conotação evolucionária, tem mostrado ótimos resultados em pacientes portadores de SM (SANTORO et al., 2003; 2008).

O presente estudo teve como objetivo analisar dois casos de pacientes portadores de SM submetidos à técnica de Santoro II, frente ao emprego de um programa de exercícios físicos aplicados um mês após a cirurgia, com duração total de nove meses. Avaliações de parâmetros biométricos, fisiológicos e bioquímicos foram analisadas neste estudo.

\section{PACIENTES E MÉTODOS}

\section{Pacientes}

Fizeram parte deste estudo dois indivíduos (um homem e uma mulher) portadores de SM, classificados de acordo com NCEP (2002), ambos pareados com 39 anos de idade. Os pacientes foram submetidos à cirurgia bariátrica Santoro II (SANTORO et al., 2003; 2008), sendo que antes da cirurgia a mulher, com 1,70 m de estatura e pesando $120 \mathrm{~kg}$, possuía IMC (Índice de Massa Corporal) de $41,53 \mathrm{~kg} / \mathrm{m}^{2}$ e o homem, com 1,80 m de estatura e $130 \mathrm{~kg}$, possuía IMC 40,13 kg/m². Este procedimento cirúrgico consiste de gastrectomia vertical, omentectomia e enterotectomia, mantendo uma porção inicial de $150 \mathrm{~cm}$ do jejuno e $150 \mathrm{~cm}$ da porção final do íleo. O termo de consentimento dos participantes do estudo foi obtido de acordo com a Resolução 196/96 do Conselho Nacional de Saúde do Brasil. Antes e depois da cirurgia, bem como durante o programa de atividade física, os pacientes foram submetidos a diferentes análises (Figura 1).

Figura 1 - Desenho experimental evidenciando os períodos de análises nas diferentes etapas do estudo ao qual foram submetidos os pacientes.

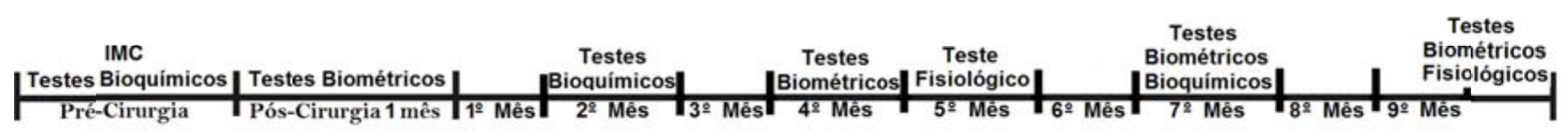


Análises antropométricas, laboratoriais e fisiológicas

Após jejum de 12 horas, uma amostra de 5 $\mathrm{mL}$ de sangue foi coletada dos pacientes para análise dos níveis de triglicérides, colesterol total, LDL e HDL, avaliados através de métodos enzimáticos. A RCQ (Relação Cintura Quadril), medida entre a circunferência da cintura dividida pela circunferência do quadril, o IMC (medida do peso corporal sobre a altura ao quadrado) e o teste fisiológico de capacidade aeróbica de Bruce ( $\left.\mathrm{VO}_{2} \max \right)$ seguiram o protocolo e a classificação da American College of Sports Medicine (ACSM, 2006).

\section{Programa de atividade física}

Após um mês de recuperação cirúrgica, os pacientes iniciaram um programa de exercícios físicos, acompanhados por uma profissional da área de educação física, durante nove meses. Os exercícios aeróbicos foram aplicados durante 30 minutos em cerca de $50 \%$ da frequência cardíaca máxima, seguidos de um treino de resistência anaeróbica e alongamentos de membros superiores e inferiores antes e após os exercícios físicos.

\section{RESULTADOS E DISCUSSÃO}

Visto que a prática de atividade física regular apresenta bons resultados em pacientes portadores de SM, levando a uma melhora das comorbidades associadas (HOPKINS et al., 2006; LAAKSONEN, 2002), foram selecionados pacientes submetidos a Santoro II para um programa de exercícios físicos regulares. A técnica cirúrgica bariátrica denominada Santoro II é um procedimento que reestrutura o sistema digestivo e que tem mostrado ótimos resultados em pacientes portadores de SM (SANTORO et al., 2003; 2008). Estudos sobre o

Figura 2 - Análise do perfil lipídico antes da cirurgia e após Santoro II associado ao programa de atividade física em dois pacientes portadores de SM. A) Colesterol; B) Triglicérides; C) LDL; D) HDL.
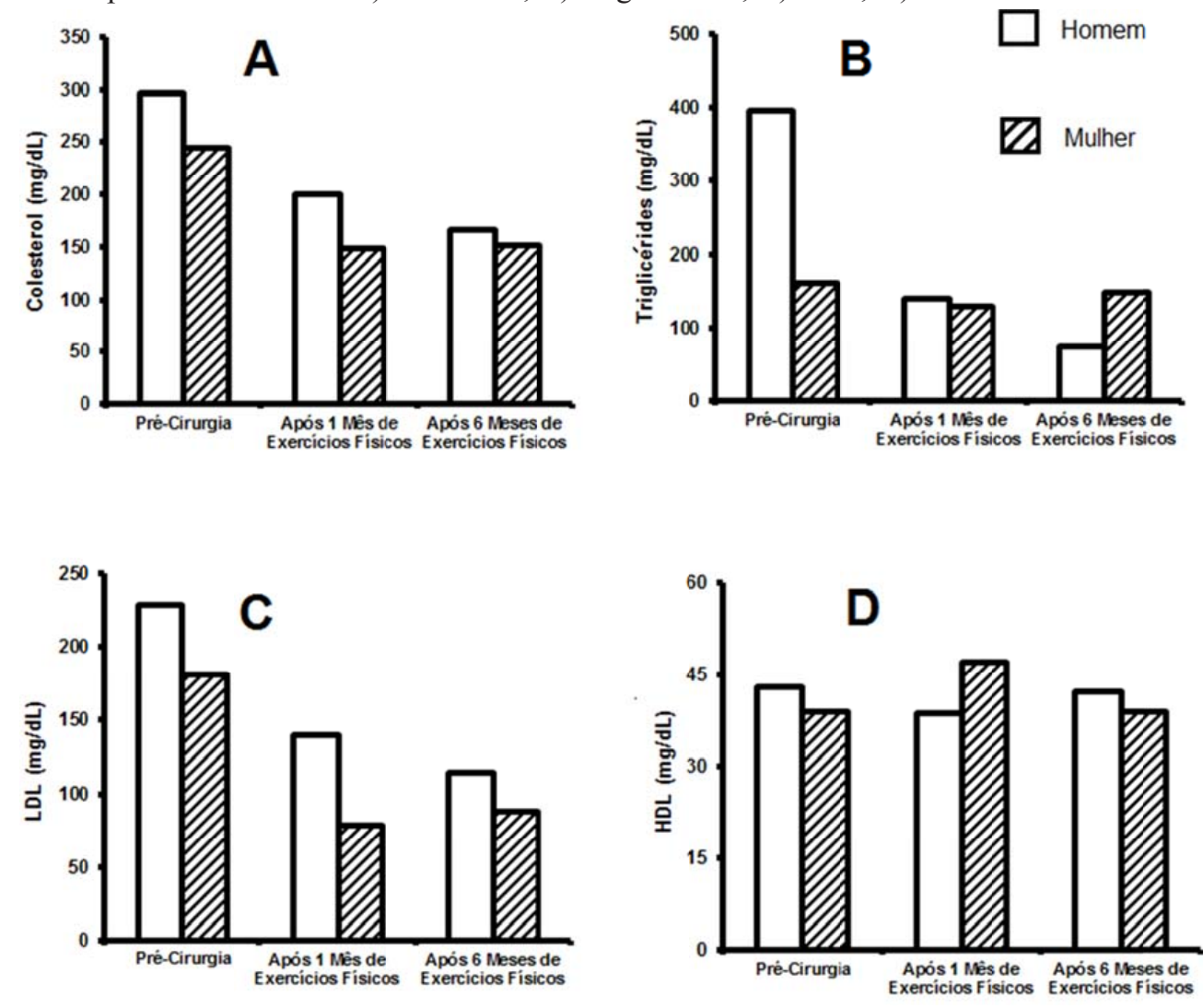
acompanhamento de pacientes após as cirurgias bariátricas, associados à prática de exercícios físicos, são escassos e extremamente importantes para o entendimento de como o organismo se comporta frente a essas intervenções.

Ambos os pacientes apresentaram melhora expressiva no perfil lipídico, transcorrido um mês do procedimento cirúrgico associado ao programa de atividade física proposto. Os resultados obtidos permitiram excluí-los dos critérios diagnósticos de pacientes portadores de SM, segundo NCEP (2002) (Figura 2). Os dados de perfil lipídico dos pacientes (colesterol total, LDL, HDL e triglicérides) foram analisados antes da cirurgia, bem como após um e seis meses da prática de exercícios físicos. Após esse período de atividades, no homem os níveis de colesterol e LDL diminuíram 43,91\% e 50,21\% respectivamente, enquanto na mulher tais valores diminuíram $38,11 \%$ e $51,38 \%$, respectivamente. Os níveis de HDL aumentaram 20,82\% na mulher após um mês do programa de exercícios físicos ser executado (38,9 para $47 \mathrm{mg} / \mathrm{dL}$ ), enquanto no homem esta variável já se encontrava normal mesmo antes da cirurgia. Nos exames subsequentes pós-cirurgia houve uma diminuição rápida dos valores totais de triglicérides de jejum, mantida com a prática de exercícios físicos. Foi observada uma melhora considerável de 81,06\% nos triglicérides circulantes no paciente masculino, enquanto a mulher teve uma redução de 8,07\%, visto que estes valores já se encontravam dentro da normalidade mesmo antes da cirurgia. De acordo com Domingues Filho (2006), a atividade física associada a uma dieta balanceada ajuda a reduzir os níveis de triglicérides.

A condição de obesidade leva a um perfil lipídico alterado com quadros de dislipidemia, como altas concentrações séricas de triglicérides, colesterol total e LDL, associado a baixos níveis de HDL, resultando riscos aumentados de doenças cardiovasculares (HU et al., 2000). Para que haja um equilíbrio entre o LDL e HDL é importante a prática regular de exercícios aeróbicos e controle alimentar, fundamentais para evitar o aparecimento de doenças cardiovasculares e outras doenças crônicas (CIOLAC et al., 2004; DOMINGUES FILHO, 2006). Segundo Pitanga (2001), o efeito da atividade física sobre o metabolismo lipídico tem sido bastante estudado nos últimos anos, sendo que o perfil lipídico é diferente entre indivíduos ativos e controles sedentários. Homens e mulheres ativos fisicamente provavelmente têm maiores níveis de HDL e menores níveis de LDL que seus pares inativos fisicamente.

Houve uma redução no IMC, em ambos os sexos, passados nove meses do programa de exercícios físicos, associada a 10 meses da cirurgia bariátrica (mulher: 36,34 para $27,34 \mathrm{~kg} / \mathrm{m}^{2}$ - redução de $41 \mathrm{Kg}$; homem: 33,1 para $26,3 \mathrm{~kg} / \mathrm{m}^{2}$ - redução de $45 \mathrm{Kg}$ ), saindo de um estágio de obesidade para sobrepeso (SMITH, 2006). As medidas da relação cintura-quadril permitiram as exclusões dos pacientes nos critérios para classificação de portadores de SM (WHO, 2000). No homem, a redução de circunferência da cintura e quadril foram $23 \mathrm{~cm} \mathrm{e}$ $15 \mathrm{~cm}$, respectivamente. Na mulher, houve a redução de circunferência da cintura e quadril de $25 \mathrm{~cm}$ e $24 \mathrm{~cm}$, respectivamente.

No teste fisiológico de capacidade aeróbica ( $\left.\mathrm{VO}_{2} \max \right)$ entre as duas avaliações do sexo feminino, obteve-se uma melhora de $1,18 \mathrm{~mL} / \mathrm{kg} /$ min entre a primeira e a segunda avaliação. Para o sexo masculino sua melhora foi de $5,03 \mathrm{~mL} / \mathrm{kg} /$ min entre a primeira e a segunda avaliação. Podemos assim confirmar que o programa de exercícios físicos apresentou resultados positivos, potencializando os efeitos da cirurgia com a diminuição do peso, consequentemente melhorando o potencial cardíaco, quando relacionado à pressão arterial e frequência cardíaca.

Ainda nos parâmetros fisiológicos podemos observar, quanto à frequência cardíaca máxima ao final do teste, que o indivíduo do sexo feminino alcançou uma frequência cardíaca de 161 
bpm (batimentos por minuto) no primeiro teste e 163 bpm no segundo teste. A recuperação cardíaca após o esforço máximo teve regressão rápida. A pressão arterial teve uma mínima de 120/70 $\mathrm{mmHg}$ e de 130/85 mmHg após esforço máximo. Estes dados podem ser considerados dentro da normalidade, principalmente em se tratando de indivíduo hipertenso controlado por medicação, de acordo com Lima et al. (2009). O indivíduo do sexo masculino também alcançou a frequência cardíaca máxima ao final do teste, sendo de 176 bpm no primeiro teste, com redução da frequência no segundo teste: 168 bpm. A recuperação cardíaca após esforço está classificada como normal, pois foi regredindo rapidamente. A pressão arterial máxima desse indivíduo após esforço foi de 130/85 mmHg e a mínima de 120/85 mmHg, classificado normal segundo Lima et al. (2009).

\section{CONCLUSÃO}

A cirurgia bariátrica é responsável por grandes melhoras em vários aspectos saudáveis do indivíduo, mas em contrapartida exercícios físicos elaborados fazem com que os percentuais de melhora sejam aumentados em todos estes aspectos. A técnica cirúrgica Santoro II, associada a um programa de exercícios físicos, mostrou ser um tratamento eficiente para indivíduos portadores de SM, não sendo relatadas complicações clínicas inerentes a este tipo de tratamento, que visa uma melhora da saúde aliada à qualidade de vida.

\section{REFERÊNCIAS}

ACSM (American College of Sports Medicine). Manual do ACSM para Avaliação da Aptidão Física relacionada à Saúde. Rio de Janeiro: Guanabara Koogan, 2006.

ALEXANDER, C. M.; LANDSMAN, P. B.; TEUTSCH, S. M.; HAFFNER, S. M. Third National Health and Nutrition Examination Survey (NHANES III); National Cholesterol Education Program (NCEP). NCEP-defined metabolic syndrome, diabetes, and prevalence of coronary heart disease among NHANES III participants age 50 years and older. Diabetes, v.52, n.5, p.1210, 2003.

CHRISTIAN, J. G.; BESSESEN, D. H.; BYERS, T. E.; CHRISTIAN, K. K.; GOLDSTEIN, M. G.; BOCH, B. C. Clinic-Based Support to Help Overweight Patients With Type 2 Diabetes Increase Physical Activity and Lose Weight. Archives of Internal Medicine, v.168, n.2, p.141-146, 2008.

CIOLAC, E. G.; GUIMARÃES, G. V. Exercício Físico e Síndrome Metabólica. Revista Brasileira Medicina Esporte, v.10, n.4, p.319-324, 2004.

DOMINGUES FILHO, L. A. Manual do personal trainer brasileiro. São Paulo: Ícone, 2006.

FORD, E. S. Prevalence of the metabolic syndrome in US populations. Endocrinology Metabolism Clinics of North America, v.33, n.2, p.333-350, 2004.

GRUNDY, S. M.; BREWER, H. B. Jr.; CLEEMAN, J. I.; SMITH, S. C. Jr.; LENFANT, C. Definition of metabolic syndrome: report of the National Heart, Lung, and Blood Institute/American Heart Association conference on scientific issues related to definition. Circulation, v.109, p.433-438, 2004.

HOPKINS, P. N.; HUNT, S. C.; WU, L. L.; WILLIAMS, G. H.; WILLIAMS, R. R. Hypertension, dyslipidemia, and insulin resistance: links in a chain or spokes on a wheel? Current Opinion in Lipidology, v.7, p.241-253, 1996.

HU, D.; HANNAH, J.; GRAY, R. S.; JABLONSKI, K. A.; HENDERSON, J. A.; ROBBINS, D. C.; et al. Effects of obesity and body fat distribution on lipids and lipoproteins in nondiabetic american indians: the strong heart study. Obesity Research, v.8, n.6, p.411-421, 2000.

ISOMAA, B.; ALMGREN, P.; TUOMI, T.; FORSEN, B.; LAHTI, K.; NISSEN, M.; et al. Cardiovascular morbidity and mortality associated with the metabolic syndrome. Diabetes Care, v.24, n.4, p. 683-689, 2001.

LAAKSONEN, D. E.; LAKKA, H. M.; SALONEN, J. T. Low Levels of Leisure-Time Physical Activity and Cardiorespiratory Fitness Predict Development of the Metabolic Syndrome. Diabetes Care, v.25, p.1612-1618, 2002.

LAKKA, H. M.; LAAKSONEN, D. E.; LAKKA, T. A.; NISKANEN, L. K.; KUMPUSALO, E.; TUOMILEHTO, J.; SALONEN, J. T. The metabolic syndrome and total and cardiovascular disease mortality in middle-aged men. The Journal of the American Medical Association, v.288, p.2709-2716, 2002.

LIMA, S. M. L.; PORTELA, M. C.; KOSTER, I.; ESCOSTEGUY, C. C.; FERREIRA, V. M. B.; BRITO, C.; VASCONCELLOS, M. T. L. Utilização de Diretrizes Clínicas e Resultados na atenção básica à hipertensão. Caderno Saúde Pública, v.25, n.9, p.2001-2011, 2009. 
NCEP (Third Report of the National Cholesterol Education Program). Expert Panel on Detection, Evaluation, and Treatment of High Blood Cholesterol in Adults (Adult Treatment Panel III). Final report. Circulation, v.106, n.3, p.143-421, 2002.

NIH Conference. Gastrointestinal surgery for severe obesity. Consensus Development Conference Panel. Annals of Internal Medicine, v.115, n.12, p. 956, 1991.

PITANGA, F. J. G. Atividade Física e Lipoproteínas Plasmáticas em Adultos de ambos os Sexos. Revista Brasileira de Ciência e Movimento, v.9, n.4, p. 25-31, 2001.

SANTORO, S.; VELHOTE, M. C. P.; MALZONI, C. E.; MECHENAS, A. S. G.; STRASSMANN, V.; SCHEINBERG, M. Digestive adaptation: A new surgical proposal to treat obesity based on physiology and evolution. Einstein, v.1, p.99-104, 2003.

SANTORO, V.; MILLÉO, F. Q.; MALZONI, C. E.; KLAJNER, S.; BORGES, P. C. M.; SANTO, M. A.; CAMPOS, F. G.; ARTONI, R. F. Enterohormonal changes after digestive adaptation: Five-Years results of a surgical proposal to treat obesity and associated diseases. Obesity Surgery, v.18, n.1, p.17-26, 2008.

SMITH, S. C.; ALLEN, J.; BLAIR, S. N.; BONOW, R.; BRASS, L. M.; FONAROW, G. C.; GRUNDY, S. M.; et al. AHA/ACC Guidelines for Secondary Prevention for Patients With Coronary and Other Atherosclerotic Vascular Disease: 2006 Update. Circulation, v.113, p. 2363-2372, 2006.

WHO. Obesity: Preventing and Managing the Global Epidemic: report of a WHO consultation on obesity. World Health Organization, p.276, 2000. 